\title{
Chance für Leistungsanbieter
}

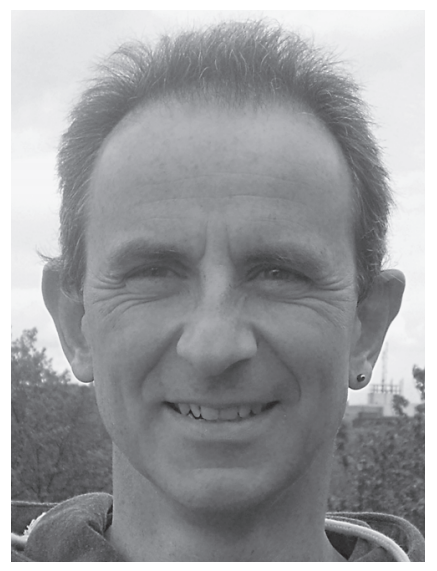

VON JÖRG ADLER

Jörg Adler ist Referent für Sozialpsychiatrie, Sucht und Behindertenhilfe beim Paritätischen Wohlfahrtsverband in SchleswigHolstein.

www.paritaet-sh.de

\author{
Mit dem Persönlichen Budget können Menschen mit \\ einer Behinderung selbst eine für sie sinnvolle Hilfe \\ einkaufen. Für Unternehmen der Sozialwirtschaft bietet \\ sich dadurch die Möglichkeit, Angebote aufzubauen, die \\ über den Sachleistungsbezug nicht refinanzierbar sind.
}

Mit der Einführung des Persönlichen Budgets wurde in Deutschland ein Paradigmenwechsel in der Behindertenhilfe eingeleitet: Teilhabe statt Fürsorge, Selbstbestimmung statt Fremdbestimmung, Freiheit statt Bevormundung das Wunsch- und Wahlrecht der Betroffenen sollte endlich in den Mittelpunkt gerückt werden.

Das Persönliche Budget ist als neue Leistungsform bereits seit dem Jahre 2001 in $\mathbb{S} 17$ Sozialgesetzbuch Neuntes Buch (SGB IX) verankert - seit dem 1. Januar 2008 besteht ein einklagbarer Rechtsanspruch. Es kann von all denjenigen beantragt werden, die eine Behinderung haben oder von Behinderung bedroht sind und einen Leistungsanspruch haben. Das Persönliche Budget umfasst alle Teilhabeleistungen sowie Leistungen der Krankenkassen, Pflegeversicherung, Unfallversicherung und der Hilfe zur Pflege im Rahmen der Sozialhilfe, soweit sie alltäglich und regelmäßig wiederkehrend erbracht werden. Sind mehrere Leistungsträger beteiligt, handelt es sich um ein »Trägerübergreifendes Persönliches Budget ", das als Komplexleistung "aus einer Hand « gewährt wird.

Die Berechtigten erhalten das Geld direkt, das bisher an einen oder mehrere Leistungserbringer als Sachleistung gezahlt wurde. Wie der Geldbetrag eingesetzt wird, ist Teil des Selbstbestimmungsrechtes und kann frei im Rahmen der getroffenen Zielvereinbarung entschieden werden. Somit stellt das Persönliche Budget eine Alternative zur Sachleistung dar.
Elf Jahre nach Einführung des Persönlichen Budgets fällt die quantitative Bilanz jedoch immer noch bescheiden aus. Die verhältnismäßig geringe Inanspruchnahme des Persönlichen Budgets liegt allerdings nachweislich nicht in dem Instrument selbst begründet. Die bisherigen Erfahrungen belegen, dass das Persönliche Budget auf jeden Fall dazu geeignet ist, die Selbstbestimmung und Teilhabemöglichkeiten für Menschen mit Behinderung zu verbessern und ihr Wunsch- und Wahlrecht zu stärken. Über 90 Prozent der Nutzerinnen und Nutzer eines Persönlichen Budgets würden sich wieder für diese Leistungsform entscheiden (so Ergebnisse der wissenschaftlichen Begleitforschung zu den Modellregionen zum Persönlichen Budget aus dem Jahre 2007 und der Studie »Implementierung des Persönlichen Budgets in Mecklenburg-Vorpommern « der Hochschule Neubrandenburg aus dem Jahre 2010).

\section{Ein verblockter Weg}

Immer noch bestimmen vor allem das Leistungssystem und die jeweiligen Institutionen die persönlichen Teilhabe- und Gestaltungsmöglichkeiten von Menschen mit Behinderung. Nicht die betroffenen Menschen selbst, sondern: Die Leistungsträger bestimmen die Inhalte der Hilfepläne und Teilhabe-Entwürfe; die Leistungsanbieter und ihre institutionellen Rahmenbedingungen dominieren den Alltag in Einrichtungen; Förderschulen sind immer noch zu sehr 
Einbahnstraßen in Richtung Werkstattleistung; Eltern lenken aus Sicherheitsgründen und aus Fürsorgegedanken den Weg ihrer behinderten Angehörigen in Einrichtungen. Folge: Menschen mit Behinderung werden nicht auf das Budget hingewiesen.

Eines der Grundprobleme des Persönlichen Budgets über alle Beteiligten hinweg ist die Frage der Haltung und des Mutes. Es ist auf fast allen Seiten immer noch nicht dazu gekommen auf breiter Basis "Erfolge mit dem Budget zu schaffen« - neue Gestaltungsräume zu entdecken und sich auf den Weg zu machen.

\section{Die Erfolgsfaktoren sind bekannt}

Betrachtet man die gelungenen Beispiele für Persönliche Budgets genauer, so stellt man fest, dass diese Menschen oft über eine gute Beratung im Hintergrund verfügen, eine Offenheit bei den Beteiligten vorhanden ist und die Menschen auf eine kompetente Begleitung in der Beantragung und Umsetzung zurückgreifen können. Sie wurden frühzeitig über die Möglichkeiten und Schwierigkeiten aufgeklärt, persönliche Ziele und Ressourcen wurden erarbeitet, die die Leistungsfähigkeit und das Umfeld der Anspruchsberechtigten einbezieht.

Diesem planmäßigen Vorgehen liegt die schlichte Erkenntnis zugrunde, dass aufseiten der Leistungsberechtigten es ganz und gar nicht selbstverständlich ist, dass sie sich der eigenen Stärken bewusst sind, ihre Interessen artikulieren und Zukunftsvisionen entwickeln, das eigene Leben weitgehend so planen und gestalten, wie man es sich wünscht. In den gelungenen Umsetzungsfällen wurden die Wünsche und Visionen der Berechtigten nicht als utopisch bezeichnet, sondern gemeinsam nach Möglichkeiten gesucht, diesen so nahe wie möglich zu kommen.

Ein weiteres Merkmal ist die schrittweise und kleinteilige Umsetzung. Es wurde nicht auf einen Schlag versucht, die gesamten Ansprüche zu verwirklichen. Dies führte zu Lernerfolgen bei allen Beteiligten.

Aus den Erfahrungen und aus der Analyse der erfolgreichen Persönlichen Budgets kann man folgern, dass Leistungsträger und Anbieter den Menschen nicht in ausreichendem Umfang Methoden und Unterstützung zur Entwicklung einer selbstbestimmten Teil- habe anbieten. Der historisch geprägte Fürsorgegedanke herrscht vor und er ist für die Träger und Anbieter der Leistung zweifelsohne der bequemere Weg. Vorschriften, Risiken und Defizitorientierung sind einfache und von fast allen Seiten akzeptierte Gründe, das Persönliche Budget für einen Leistungsberechtigten nicht in Erwägung zu ziehen oder abzulehnen. Starre Leistungssysteme, Informationsdefizite, mangelnde Flexibilität und Fantasie prägen einen Teil de Hintergrundes.

In den europäischen Ländern, in denen das Persönliche Budget eine nennenswert hohe Zahl von Nutzern verzeichnet (z. B. Holland), existieren kompetente Unterstützungsstrukturen, die über Methoden der Zukunftsplanung verfügen, Zeit und Ressourcen haben, um die Menschen in allen zusammen-

\section{Chancen für Leistungsanbieter}

Trotz aller Hürden steigen die Zahlen der Budgetnehmerinnen und Budgetnehmer an, weil die Menschen eine unabhängige, auf ihre Bedürfnisse zugeschnittene Unterstützung suchen, die es ihnen erlaubt ihr Leben weitgehend oder zumindest in großen Teilen frei und selbstbestimmt zu gestalten.

Diese Menschen können ihren Lebensstil, ihren Tagesrhythmus, ihre Gewohnheiten leben, wie andere Menschen auch. Sie können die Kindergärten, Schulen, zum Teil Arbeitsstätten und andere Einrichtungen nutzen und tauchen im öffentlichen Leben auf. Sie können sich teilweise die Behandlungen und Therapien suchen, auf die sie am besten reagieren. Es ist zu beobachten, dass viele Menschen sich mit Unterstützern

\section{"Der Aufbau von Angeboten für das Persönliche Budget kann neue Geschäftsfelder erschließen"}

hängenden Fragen zu beraten und zu begleiten. Meist sind diese Strukturen von betroffenen Menschen und deren Verbänden selbst aufgebaut worden. Sie refinanzieren sich aus den Budgets.

Auch in Deutschland versuchen die Betroffenen und ihre Verbände, diese Netzwerke zu knüpfen. Sie sind dabei aber sehr auf sich allein gestellt und erfahren zu wenig Unterstützung von Leistungs- und Rehabilitationsträgern, Leistungsanbietern und anderen. Sie können kaum auf finanzielle Förderung zurückgreifen und die Refinanzierung aus den Persönlichen Budget ist in den seltensten Fällen möglich, da das deutsche Recht dem - zurzeit noch - entgegensteht.

Leider ist auch zu beobachten, dass die Kompetenz, die eine Umsetzung eines umfänglicheren Budgets oder ein Budget nach dem Arbeitgebermodell erfordert, in den seltensten Fällen bei Ämtern und Anbietern anzutreffen ist. Nach der Bewilligung folgt die schwierige Suche nach kompetenten Assistenzkräften für die Leistungserbringung oder deren umfangreiche Einarbeitung, weil dafür bisher keine Infrastruktur aufgebaut wurde. ihre eigenen, selbstbestimmten Wohnund Unterstützungsleistungen aufbauen. Sie tun dies, weil sie keine passgenauen Angebote "am Markt« finden.

Hier liegt die Herausforderung für die Einrichtungen und Dienste, diese geforderte Unterstützung qualifiziert und zugleich personenzentriert zu gestalten. Hierin bietet sich die Chance, in der Region als Leistungsanbieter aufzutreten, der Menschen mit Behinderungen in ihrem Sinne unterstützt und gesetzliche Vorgaben qualitativ hochwertig mit Leben füllt und deshalb zu vermehrter Nachfrage führt.

Der Aufbau von Angeboten für Budgetnehmende kann für Einrichtungen langfristig gesehen die Chance sein, neue Geschäftsfelder zu erschließen. So können beispielsweise Unterstützungsund Therapieformen, die im Sachleistungssystem in der Regel nicht oder nicht mehr finanziert werden, möglicherweise über das Persönliche Budget umgesetzt oder fortgeführt werden. Die Ausgestaltung von Dienstleistungen bestimmt in Zukunft nicht mehr allein der Leistungsträger, sondern vor allem das Bedürfnis der Budgetnehmerinnen und 
Budgetnehmer. Von den Leistungsanbietern sind also kreative Ideen und flexible Lösungen bei der Umsetzung gefordert und eine eindeutige Positionierung für die Belange der Betroffenen.

Bereits heute ist - nicht nur beim Persönlichen Budget - der Trend zu erkennen, dass Empfänger sozialer Dienstleistungen mehr Einfluss auf die Auswahl der Angebote und Anbieter erhalten. Einrichtungen müssen in der Folge sich selbst und ihre Angebote so präsentieren, dass ihre Leistungen auch nachgefragt werden. Marketing-Maßnahmen können helfen, sich gegenüber anderen Anbietern abzugrenzen und ein eigenes, möglichst unverwechselbares Profil zu entwickeln. Die Entscheidung, in $\mathrm{Zu}$ kunft Leistungen für Kundinnen und Kunden mit einem Persönlichen Budget anzubieten, könnte ein Element solch eines Profils sein.

\section{Resümee}

Das Persönliche Budget wird sicherlich noch brauchen, sich in Deutschland mit einem nennenswerten Prozentsatz von Nutzern durchzusetzen. Es fehlen Beratungs-, Unterstützungs- und Begleitungsstrukturen. Es mangelt an Kompetenz bei Mitarbeitenden, es fehlt an Information und Motivation bei den Betroffenen, um die Beantragung eines Persönlichen Budgets zu wagen.

Trotzdem bietet es schon heute trotz aller Hürden und Hemmnisse für die Menschen mit Behinderungen eine variable und gestaltungsoffene Möglichkeit, ein selbstbestimmtes Leben zu führen. Soziale Organisationen und Unternehmen, die sich auf die UN Behindertenrechtkonvention berufen und die sich in ihren Leitbildern einer personenzentrierten Versorgung verpflichten, die aktiv an ein selbstbestimmtes Leben von Menschen mit Behinderungen fördern wollen, sollten sich mit dem Persönlichen Budget auseinandersetzen und es fördern.

Das Persönliche Budget bietet die Chance, den Menschen von der Frühförderung bis zur Gestaltung ihres Lebensabends zu einer aktiven Teilhabe am Leben zu verhelfen und gleichzeitig sinngefüllte Arbeitsplätze zu schaffen.

\section{Beispiel Leistung »Bundesligaspiel»}

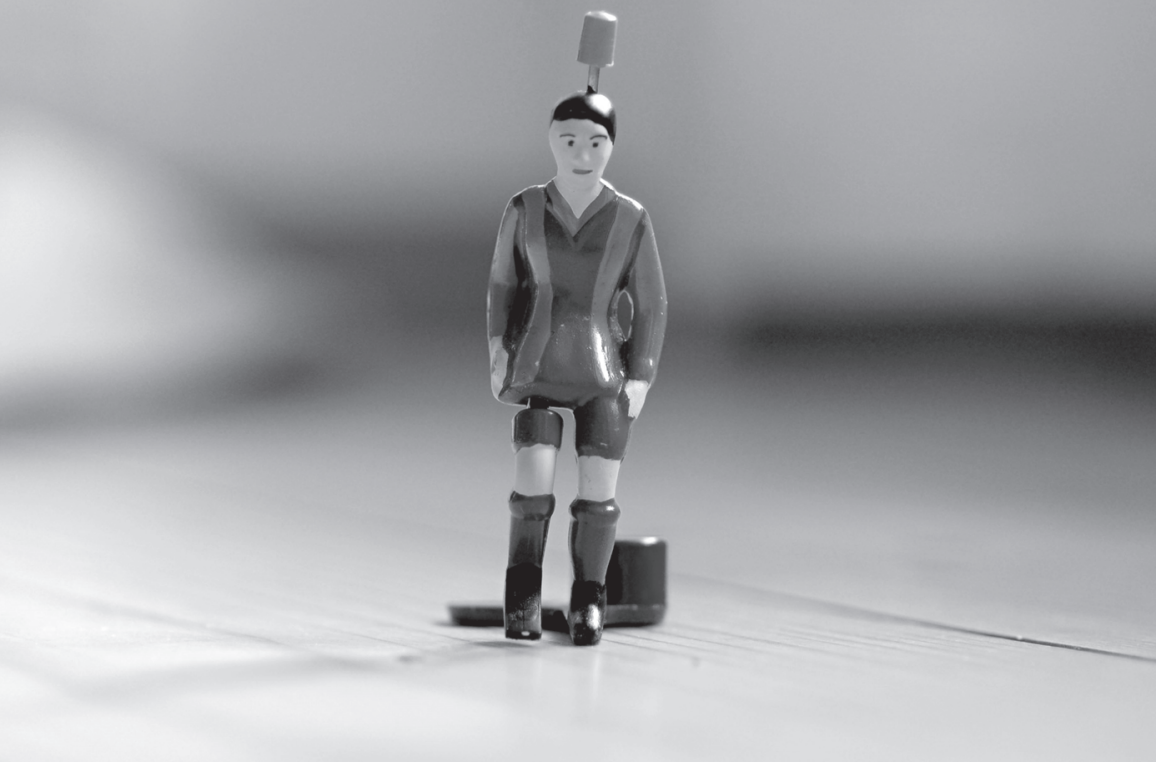

Ein Leistungsberechtigter möchte ein Bundesliga-Fußballspiel besuchen. Dies soll aus Sicht der pauschalen Tagessatzfinanzierung und aus Sicht des Persönlichen Budgets betrachtet werden:

- Tagessatzfinanzierung: Nach traditioneller Leistungsbeschreibung zählt der Besuch eines Fußballspiels zu den sozialen Aktivitäten. Der oder die Sozialpädagoge/-in und der Praktikant fahren mit dem oder der Leistungsberechtigten und zwei weiteren Betreuten im Kleinbus der Einrichtung ins Stadion. Insgesamt dauert dies mit Vorbereitung und Stau beim Hin- und Herfahren fast fünf Stunden und wird pauschal über den Tagessatz der Leistungsberechtigten finanziert.

- Persönliches Budget: Im Rahmen eines Persönlichen Budgets wird kein Leistungsberechtigter bereit sein, abstrakt für "Soziale Betreuung " fünf Stunden Betreuung zu finanzieren. Wie soll der oder die Budgetnehmer/-in einsehen, dass er oder sie für 90 Minuten (zumindest anteilig) fünf Fachleistungsstunden bezahlen soll? Das Risiko für die lange Dauer kann kaum einseitig bei den Leistungsberechtigten liegen. Demnach sind die Hin- und Rückfahrt (Art der Begleitung, Verkehrsmittel) eigene zu beschreibende Leistungen, ebenso ist der Betreuungsumfang während des Spiels nach Art und Umfang zu beschreiben (Plätze, Pausen, Verköstigung, Krisenintervention). Angebotsbeschreibungen werden sich also in eine Richtung entwickeln, wie wir sie beispielsweise aus Katalo- gen für Pauschalreisen kennen. Damit sind aber beide Parteien nicht mehr im "weichen " Lager sozialhilferechtlicher Dreiecksverhältnisse, sondern in der »harten« Welt bürgerlichen Vertragsrechts und umfangreicher zivilrechtlicher Haftungsrisiken für die Leistungsanbieter.

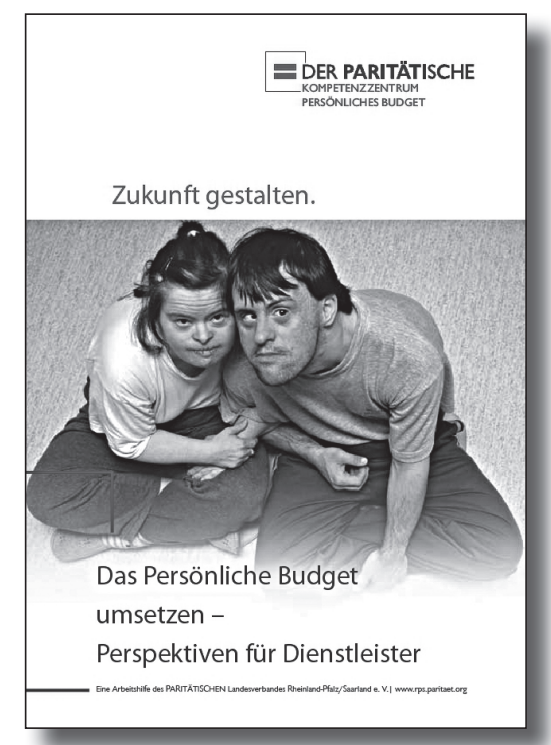

Quelle: Kompetenzzentrum Persönliches Budget (Hg.): Zukunft gestalten. Das Persönliche Budget umsetzen. Perspektiven für Dienstleister. Die 58-seitige Broschüre mit Arbeitshilfen und Vertragsmustern kann kostenlos aus dem Internet heruntergeladen werden: http://www.budget. paritaet.org/?id=2307 